\title{
Quadriceps Muscle Strength Exercises and Its Effect on Osteoarthritis Patients at Aswan University Hospital
}

\section{Leila Ahmed Abdu; Amal Mohamed Ahmed; Marwa Mohammad Abd elbaky}

\author{
1. B s.C in Nursing, Faculty of Nursing - Aswan University \\ 2. Professor of Medical Surgical Nursing, Dean of Faculty of Nursing - Aswan University \\ 3. Assistant Prof. of Medical Surgical Nursing (Critical care nursing), Faculty of Nursing - Minia University
}

\begin{abstract}
Background: Osteoarthritis is the most common form of arthritis among adults, leading to substantial pain and disability. Aim: Evaluate the effect of quadriceps muscle strength exercise on the patients with osteoarthritis at Aswan university hospital. Research Design: is Quasi experimental. Subjects \& Setting: A convenient sample of (60) newly diagnosed osteoarthritis of the knee joint adult patient recruited from the physical medicine department and rehabilitation department at Aswan university hospital divided into two groups study and control. Tools: two tools, tool one Patient's General Assessment Sheet; and The Knee Osteoarthritis Assessment checklist. Tool two is Algo functional for Knee Osteoarthritis Index (AKOI). Results: $76.7 \%$ of study and $60 \%$ of the control group were female. This study revealed statistically significant difference between study and control group of Knee Osteoarthritis Assessment post quadriceps muscle strength exercises regarding complete flexion and complete extension of knee, $\mathrm{p} \leq 0.05$. There was improvement in mean and S.D of pain in the study group than the control after applying the exercise at the $3^{\text {rd }}$ and $6^{\text {th }}$ week. Improvement in mean and SD between study and control group regarding all items of Maximum distance walked during follow up at $3^{\text {rd }}$ and $6^{\text {th }}$ week and $p \leq 0.05$. Conclusion: quadriceps muscle strength exercise effective in reduce pain and improve movement. Recommendations: Nurses should emphasis to provide a quadriceps muscle strength exercises on the patients with osteoarthritis.
\end{abstract}

Key Words: Exercises, Osteoarthritis, Patients, Quadriceps Muscle \& Strength.

\section{Introduction}

Osteoarthritis (OA) is the most common musculoskeletal disease in the world, affecting the synovial joints Saad et al., (2015). It is no longer considered to be a normal part of the aging process, but growing older continues to be consistently identified as one risk factor for disease development. Cartilage destruction can actually begin between ages 20 and 30 years, and the majority of adults are affected by age 40. Osteoarthritis (OA) is a common chronic condition resulting in pain, fatigue, functional limitations, increased healthcare utilization and high economic costs to society Litwic et al., (2013).

Pain is the first and predominant symptom, causing loss of movement ability and often stiffness. "Pain" is generally described as a sharp ache, or a burning sensation in the associated muscles and tendons. The pain is intermittent and is worse with joint use and better with rest. Because there is no cure for osteoarthritis, collaborative management are directed towards controlling symptoms, maintaining and improving joint mobility, increasing level of activity, reducing physical disability, handicap, improving health-related quality of life, limiting the progression of joint damage, and educating patients about the nature of the disorder and its management. Treatment modalities of OA include a combination of the following elements; pharmacological management, physical exercise therapy, weight control, joint protection, and surgical management Ahamed (2014).

Several published guidelines currently recommend non-pharmacological interventions as a first line of treatment for knee OA. Nurses play an important role in patient rehabilitation. They should inform patient about proper application of exercise to improve muscle strength, joint flexibility and cardiorespiratory fitness. Patients may require cues as well as verbal instructions, and illustrated pictures,

P a g e | 118 particularly in the early phases of exercise program. It is important that these patients use proper body mechanics and body alignment. Nurses also follow up their patient's during and after any exercise session, the nurse should assess for any signs of excessive joint strain, presence of pain during activity, lasting more than 1 to 2 hours after exercise, swelling, fatigue, and weakness. If joints hurt or redness or swellings are noticed, then patient is advised to try a little exercise. A warm bath to soothe aching muscles and joints after a workout is important as well as monitoring feelings of patient after exercise (Teixeira, 2012).

\section{Significance of the study}

Knee osteoarthritis (OA) is a common chronic and progressive degenerative joint condition that contributes greatly to disability in the general population. Knee osteoarthritis is characterized by pain, stiffness, decreased range of motion, and muscle weakness. These symptoms can limit the ability to climb stairs, rise from a chair, and walk, causing dependency in daily activities. World health organization (WHO) estimated that knee OA account for $83 \%$ of total population due to increased longevity, reduced physical activity levels, and increasing prevalence of obesity (WHO, 2013). OA as a musculoskeletal disease it affects approximately 27 million Americans. It is anticipated that OA will become the eighth most important cause of disability in men and the fourth most important cause of disability in women according to the World Health Organization (WHO) Saad et al., (2015). In Egypt, more than five million people have OA Sebbag et al., (2019).

The goal of nursing management is helping patient to develop a pain management protocol that maximizes comfort and functional abilities. Nursing goal also directed to help patient adapt to this chronic illness by teaching methods that

Leila A., et al 
minimize pain and stiffness, increase mobility prevent deformity and preserve normal family and social role interaction. Therefore, the present study had carried out in an attempt to evaluate the effect of exercise protocol of osteoarthritis patient at Aswan University Hospital as indicated by improvement of patient's knowledge and practices related to osteoarthritis, relive of joint pain and preventing joint stiffness.

\section{Aim of the Study}

Evaluate the effect of quadriceps muscle strength exercise on the patients with osteoarthritis at Aswan university hospital.

\section{Research Hypothesis}

To fulfill the aim of the present study the following hypothesis were formulated:

1- Patients of the study group whom received quadriceps muscle strength exercises will have improvement in joints function than the control group.

2- Patients of the study group whom received quadriceps muscle strength exercises will have better assessment of The Knee Osteoarthritis Checklist than the control group.

Patients of the study group whom received quadriceps muscle strength exercises will have higher mean and S.D of the Algo functional for Knee Osteoarthritis Index than those of the control group whom received the routine hospital nursing care

\section{Study procedure}

Preparatory phase

Research design: is a quasi-experimental study design.

Setting: This study was carried out at the physical medicine and rehabilitation department at Aswan university Hospital.

Sample: A purposes sample of sixty adult patients from both sexes were collected conveniently in the study and devided equally to two groups (30 patients each).

\section{Sample equation:}

The sample size was selected according to the following formula:

$$
\begin{aligned}
N & =\frac{t^{2} \times p^{(1-p)}}{m^{2}} \\
N & =\frac{(1.96)^{2} \times 0.04(1-0.04)}{0.05^{2}} \\
N & =60 \text { patients }
\end{aligned}
$$

\section{Description:}

$\mathbf{N}=$ required sample size

$\mathbf{T}=$ confidence level at95\% (standard value of 1.960 )

$\mathbf{P}=$ estimated prevalence of patient with knee osteoarthritis at

Aswan University Hospital 2018 (0.040)

$\mathbf{M}=$ margin of error at $5 \%$ (standard value of 0.050$)$.

\section{Inclusion criteria:}

Patients of the study met the following criteria: -

A. Mild and moderate osteoarthritis of the unilateral or bilateral knee joint

B. Age from 18 years up to 65 years.
C. Have not been involved in scheduled program of regular exercises or strength training within the last 6 months.

D. Have not been received intra -articular steroid injections within the previous three months.

\section{Study duration:}

The study was conducted in a period of six months (from July 2019 to the end of December 2019).

\section{Tools of the study}

The tools used in the study were two.

Tool (I): Patient's General Assessment Sheet: this tool was developed and applied by the researcher after a reviewing the related literature it includes three parts:

Part one: include patient's socio demographic data as, patient's name, age, sex, level of education, marital status, perceived economic status, and occupation.

Part two: include patient general assessment as medical diagnosis, body mass index, gait stability, and using assistive device for ambulation or not.

Part three: The Knee Osteoarthritis Assessment Checklist. This checklist was developed and applied by the researcher after reviewing of literature (Saad, 2015) to assess:
A. Tenderness, edema and shape of both knee joints.
B. Joint stiffness
C. Knee joint effusion.
D. Crepitation.
E. Temperature of the skin around the knee joint.
F. Range of motion of both knee joints was measured by a Goniometer (a device that measures movement of joint in degrees). The normal knee flexion is $(0$ - $\left.135^{\circ}\right)$, and the normal knee extension is $\left(0-15^{0}\right)$.

\section{Tool (II): The Algo functional for Knee Osteoarthritis Index (AKOI) \\ This index was adopted from (Lequene, 1998). It} was used to evaluate pain or discomfort of the affected joint, maximum distance walked tolerance and activities of daily living. It included three main parts:

Part one: This part was used to evaluate pain or discomfort of the affected joint using the pain section of the Algo functional osteoarthritis index. It contains five questions concerning the severity of pain during various activities, each question scored $0-2$. Patient was asked to grade level of pain overall rather than defining site of pain, patients with higher scores indicating more severe pain.

Part two: this part was used to evaluate maximum distance walked scoring system ranged from 0-8. Patients with higher scores indicating more disability and lower scores indicating better function.

Part three: This part was used to assess physical function of the joint during activities of daily living. It contained four questions and scores will summated to produce a global function score, ranging from $0-2$. Score 0 indicates inability to perform activities of daily living, whereas score 2 Indicates perform activities of daily living without difficulty. 


\section{Validity and Reliability}

Tools of the study were submitted to five experts in the field of Medical-Surgical Nursing and rehabilitation for content validity, completeness, and clarity of the items and the necessary modification were done thereafter.

- The reliability of the tools was tested using the Cronbach alpha coefficient and it was (90\%).

\section{Pilot study}

- A pilot study was conducted on $10 \%$ (6) of patients to examine the feasibility of the study. According to this pilot study, the required modifications were made.

\section{Ethical consideration}

- An official permission was obtained from the head of Aswan University Hospital \& physical medicine and rehabilitation department to conduct the study.

- Oral consents were obtained from the patients after explaining the aim of the study and assuring them complete confidentiality and that they can withdraw at any time of the study.

\section{Implementation phase}

- Patient's interviews were carried out individually for both groups to collect sociodemographic data and general assessment using the tool 1 at the outpatient clinic, which took approximately 20-30 minutes for each interview.

- An initial assessment was done by the researcher for both groups in the affected knee joint at the first visit to obtain baseline data used in the comparison using part three tool I and tool II.

- Physical examination of the affected joint was done by the researcher for both groups using part three tool I, which included-

A. Tenderness, edema and shape of both knee joints.

B. Joint stiffness

C. Knee joint effusion according to the following: 1st degree: Loss of the normal concavity medial to the patella.

2nd degree: There is sufficient fluid present to float the patella of the under-ling femur.

3rd degree: A tense effusion preventing full extension of the knee.

D. Crepitation.

E. Temperature of the skin around the knee joint.

F. . Range of motion of both knee joints was measured by a Goniometer (a device with two arms revolving around an axis centered on the medial side of the knee joint. It measures movement of joint in degrees) ROM is measured by having the patient move the leg from maximal extension to maximal flexion. The movable arm of the goniometer indicates the angle in degrees. The normal knee flexion is $\left(0-135^{\circ}\right)$, and the normal knee extension is $\left(0-15^{0}\right)$.

- The exercise protocol was developed based on a thorough review of related literatures. It was then designed to be introduced to the study groups patients in Arabic.

- The exercise protocol was carried out by the researcher for each patient in the study groups individually. This protocol consisted of stretching exercise, strengthening exercises and range of motion exercise. Clear and simple instructions were offered to each patient, before exercise training protocol. Each patient had an individualized exercise prescription with pictures that contained the goal of the exercise, the type of movement in each exercise, the frequency with which the exercise should be performed and the duration. Each patient was asked to re demonstrate the exercises until the patient gain the skills to perform these exercises, correctly and actively, at home.

- Exercise protocol consists of 3 phases: warm-up, muscle strength period and a cool-down period.

\section{A. Warm-up period:}

After selection of patient according to the inclusion criteria, the warm-up period: This was the first step which increase blood flow into the involved muscles and elevate muscular temperature, were performed for 5 to $15 \mathrm{~min}$ before engaging in the main exercise. Clear and simple instructions were offered to each patient in the study groups before exercise training protocol. Each patient have an individualized exercise prescription with pictures that contained the goal of the exercise, the type of movement in each exercise, the frequency with which the exercise should be performed and the duration. Each patient was asked to re demonstrate the exercises until the patient gain the skills to perform these exercises, correctly and actively, at home. Performing warmups can lower the risk of injuries in the muscles and tendons, as well as reduce heavy loads on the heart, which can occur when high-intensity exercises are suddenly started. Motivation of patient during this period was done through psychological support.

\section{B. Muscle strength training period: -}

This is the stage following the warm up period. It is improving the physical function, alleviating pain it can be described as a dynamic, repetitive exercise requiring the use of large muscle groups.

The patients were divided in to two groups control group (30) patient and study group (30) patient The experimental group performed exercises, whereas the control group did not performed any exercise program but they were received the routine nursing care. The schedule of muscle strength training exercises was done by the researcher three times per week for 3 weeks. In each session, muscle strength exercises was performed by the researcher to each patient. Muscle strength exercises consist of 10 sessions. The researcher carried out the physical exercise protocol individually for each patient. Patients were instructed to perform the following set of exercise every day for 6 weeks. All exercises were performed once a day for the 1 st week, and these progressed to twice a day until the 3rd week and three times a day until the 6th week. Patients were instructed to repeat every exercise 10 times

\section{A-Stretching Exercises:}

These exercises involved the calf and hamstring muscles. For a standing calf stretch: the patient stands with the heel of the foot on the ground behind the patient, the toes point straight ahead. The patient leans forward until a moderate pull is perceived in the calf musculature. The patient may use his or her arms for support against a wall or furniture as needed. The patients were instructed to hold for 30 seconds. For hamstrings muscle stretch: the patient lies in a supine 
position with the lower extremity maintained as straight as possible, the hip is flexed to $90^{\circ}$, the knee is straightened and the proximal lower leg supported by the hands until a moderate pull is perceived in the posterior thigh and calf and the foot should be dorsiflexed. The patients were instructed to hold for 30 seconds.

\section{B- Strengthening exercises:}

These exercises involved statis quadriceps sets in knee extension, straight leg raise, partial squats weight-lessened with arm support as needed, step-ups and isometric hip adduction.

\section{Statis quadriceps sets in knee extension}

The patient lies in a supine position supported on elbows with the knee in full extension. Patient is instructed to contract the quadriceps muscle and push the knee down while maintaining the foot in full dorsiflexion. The patients were instructed to hold each contraction for $6 \mathrm{sec}$ with a10- seconds with rest between repetitions.

\section{Straight leg rise:}

The patient lies in a supine position. They were instructed to perform a maximum quadriceps contraction prior to the lifting phase of exercise. Then they were instructed to lift the leg up to $10 \mathrm{~cm}$ above the plinth. The patients were instructed to hold each contraction for 10 seconds.

\section{Partial squats weight-lessened with arm}

Support as needed: the patient performs a partial squat, keeping the knees centered over the feet, return to standing by contracting the quadriceps and gluteal muscles. The patients were instructed to hold each contraction 30 seconds with hips and knees as straight as possible, repeat for 30 seconds and progress to full body weight without support and additional bouts.

Step-ups: the patient stands in front of a low step; places foot of involved leg on step and bring body over foot to stand on the step, use as little push off assistance from the contralateral foot as possible and step down with the contralateral foot. The patients were instructed to repeat for 30 seconds and progress by increasing the height of the step and additional bouts. Alternate legs if both knees are involved.
Isometric hip adduction: the patient lies in a supine position. A small pillow was put between the knees. The patients were instructed to perform isometric hip adduction exercise while pressing the pillow between the knees and maintain the adduction with contraction for 5 seconds.

\section{C-Range of Motion Exercises:}

Range of motion exercises were performed for both legs.

\section{Knee in mid-flexion to full-extension:}

The patient is positioned supine or supine supported on elbows, Knee is brought to $45^{\circ}$ of flexion with the foot sliding on the surface that the patient is lying on. The knee is then fully extended with a strong quadriceps muscle contraction. Patients were asked to repeat two 30-second bouts with 3second hold at end range

\section{Knee in mid-flexion to full-flexion:}

The patient is positioned supine or supine supported on elbows, the knee is brought to full flexion with assistance of the upper extremities or a strap. A gentle challenge to endrange flexion is sustained. Patients were asked to repeat two 30 -second bouts with 3 -second hold at end range.

\section{Cool-down period:}

Cool down period is the third component of the exercise. In this period after the patient was performing the period of quadriceps muscle strength, the patient asked to take 5 to 8 minutes cool down period. Through this time exertion is decreased to a low intensity cooling down allows the heart rate to return to its resting rate.

- The patients of both groups were followed up weekly in the outpatient Clinic for six weeks to ensure patients compliance to the instructions given using part three tool I and tool II.

\section{Evaluation phase:}

- Evaluation of pain, rang of motion of knee joint (flexion, extension), maximum distance walked and activities of daily living was done by the researcher using part three tool I and tool II at the $3^{\text {rd }}$ week and $6^{\text {th }}$ week post exercise protocol to determine the effects of the exercise on the affected knee joint for each patient.

Table 1: Frequency distribution of patient's socio-demographic and medical data for studied patient's (study and control) number (60)

\begin{tabular}{|c|c|c|c|c|c|}
\hline \multirow{2}{*}{ Variables } & \multicolumn{2}{|c|}{ Study group $=\mathbf{3 0}$} & \multicolumn{2}{|c|}{ Control group $=30$} & \multirow[t]{2}{*}{ p.v } \\
\hline & No. & $\%$ & No. & $\%$ & \\
\hline \multicolumn{6}{|c|}{ Age by years } \\
\hline $18-<25$ years & $\mathbf{0}$ & 0.0 & 1 & 3.3 & \multirow{4}{*}{$.271 \mathrm{~ns}$} \\
\hline $25-<35$ years & 3 & 10.0 & 7 & 23.3 & \\
\hline $35-<45$ years & 12 & 40 & 7 & 23.3 & \\
\hline More than 45 & 15 & $\mathbf{5 0 . 0}$ & 15 & 50.0 & \\
\hline \multicolumn{6}{|c|}{ Sex } \\
\hline Male & 7 & 23.3 & 12 & 40.0 & \multirow{2}{*}{$.267 \mathrm{~ns}$} \\
\hline Female & 23 & 76.7 & 18 & 60.0 & \\
\hline \multicolumn{6}{|c|}{ Level of education } \\
\hline illiterate & 1 & 3.3 & 6 & 20.0 & \multirow{6}{*}{$.274 n s$} \\
\hline Read and write & 1 & 3.3 & 1 & 3.3 & \\
\hline Primary school & 4 & 13.3 & 7 & 23.3 & \\
\hline Preparatory school & 10 & 33.3 & 7 & 23.3 & \\
\hline Secondary school & 6 & 20.0 & 5 & 16.7 & \\
\hline university & 8 & 26.7 & 4 & 13.3 & \\
\hline \multicolumn{6}{|c|}{ Unilateral Knee osteoarthritis (OA) } \\
\hline Right & 8 & 26.7 & 7 & 23.3 & $.559 \mathrm{~ns}$ \\
\hline
\end{tabular}


Minia Scientific Nursing Journal (Print - ISSN 2537-012X) (Online - ISSN 2785-9797) Vol. (9) No. (1) June 2021

\begin{tabular}{|c|c|c|c|c|c|}
\hline \multirow{2}{*}{ Variables } & \multicolumn{2}{|c|}{ Study group =30 } & \multicolumn{2}{|c|}{ Control group $=30$} & \multirow[t]{2}{*}{ p.v } \\
\hline & No. & $\%$ & No. & $\%$ & \\
\hline Left & 12 & 40.0 & 9 & 30.0 & \\
\hline \multicolumn{5}{|l|}{$\begin{array}{l}\text { Bilateral Knee } \\
\text { Osteoarthritis }\end{array}$} & \\
\hline Right and left & 10 & 33.3 & 14 & 46.7 & \\
\hline \multicolumn{5}{|l|}{ Osteoarthritis degree } & \multirow{3}{*}{$.150 \mathrm{~ns}$} \\
\hline Mild & 8 & 26.7 & 4 & 13.3 & \\
\hline Moderate & 22 & 73.3 & 26 & 86.7 & \\
\hline \multicolumn{5}{|l|}{ Gait } & \multirow{4}{*}{$.118 n s$} \\
\hline Stable & 6 & 20.0 & 13 & 43.3 & \\
\hline Limping & 18 & 60.0 & 11 & 36.7 & \\
\hline Dragging & 6 & 20.0 & 6 & 20.0 & \\
\hline
\end{tabular}

Table 1: Show that women are more affected and burdened by osteoarthritis of the knee than men. It is Show that $50.0 \%$ of the study and control group were aged more than 50 years old. As regards sex it was observed that the majority of the studied sample, $76.7 \%$ of study and $60 \%$ of the control group were female respectively, $33.3 \% \& 23.3 \%$ of the study and control group were preparatory educated. Concerning the affected limb, the left leg account for $40 \%$ of the study group and $30 \%$ in the control group the patient with both legs account for were $33.3 \%$ of the study and $46.7 \%$ of the control group. Regarding gait, more than half $60 \%$ of the study group had Limping compared to $36.7 \%$ of the control group. There are no statistical differences between study and control group regarding all items of demographic and medical data

Table 2: Comparison between the study and control groups regarding items of the Knee Osteoarthritis Assessment Checklist between $n=60$

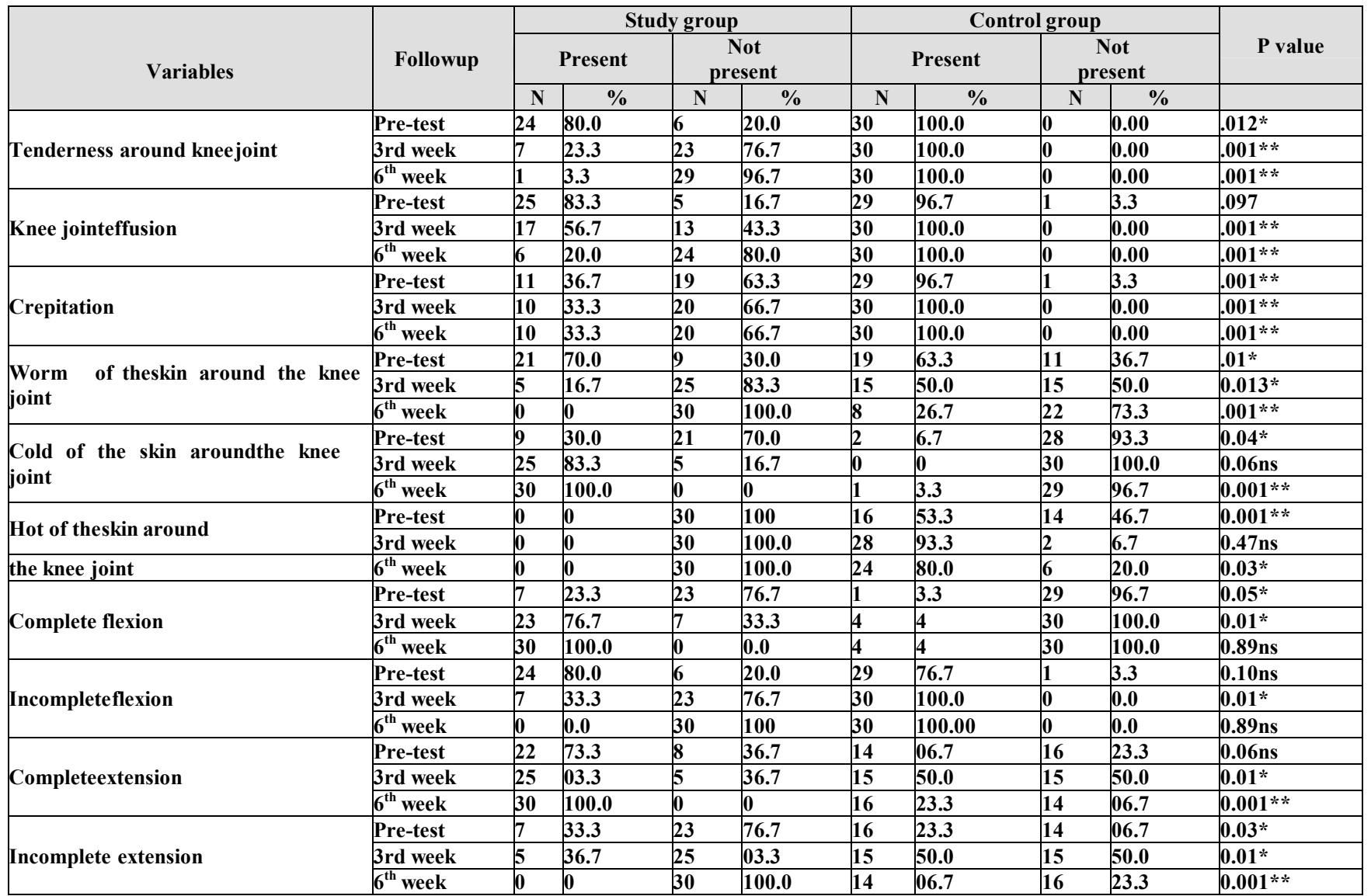

\section{Chi-Square Tests Ns= Non significant difference $\mathbf{P}>\mathbf{0 . 0 5}$}

Table 2: reveals that $80.0 \%$ of the patients in study group have tenderness around knee joint at the pretest compared with $100.0 \%$ in control group which changed into $23.0 \%$ in study group after 3 week of intervention compared with $100.0 \%$ in control group and into $3,3 \%$ in study group after 6 week of intervention compared with $100.0 \%$ in control group with highly significant difference between them $\mathrm{p} \leq 0.05$.

It also showed that $83.3 \%$ of the patients in study group complain of knee joint effusion at the pretest compared with $96.7 \%$ in control group which changed into $56.7 \%$ in study group after $3^{\text {rd }}$ week of intervention compared with $100.0 \%$ in control group and into $20.0 \%$ in study group after $6^{\text {th }}$ week of intervention compared with $100.0 \%$ in control group with highly significant difference between them $\mathrm{p} \leq 0.05$.

Regarding to crepitation it was reported that $36.7 \%$ in study group in pretest compared with $96.7 \%$ in control group which changed into $33.3 \%$ in study group after 3 week of intervention compared with $100.0 \%$ in control group and into $33.3 \%$ in study group after 6 week of intervention compared with $100.0 \%$ in control group, with highly significant difference between them $\mathrm{p} \leq 0.05$. 
Minia Scientific Nursing Journal (Print - ISSN 2537-012X) (Online - ISSN 2785-9797) Vol. (9) No. (1) June 2021

Table 3: Comparison between study and control groups regarding all items of Algo functional for Knee Osteoarthritis Index

\begin{tabular}{|c|c|c|c|c|c|c|c|c|c|c|c|c|c|c|c|c|}
\hline \multirow{3}{*}{ Items } & \multirow[b]{4}{*}{ None or insignificant } & \multicolumn{6}{|c|}{ Study group $=\mathbf{3 0}$} & \multicolumn{6}{|c|}{ Control group $=\mathbf{3 0}$} & \multirow[b]{2}{*}{ P1 } & \multirow[b]{2}{*}{ P.2 } & \multirow[b]{2}{*}{ P.3 } \\
\hline & & \multirow{2}{*}{\multicolumn{2}{|c|}{\begin{tabular}{|l} 
Pre-test \\
N (\%)
\end{tabular}}} & \multirow{2}{*}{\multicolumn{2}{|c|}{\begin{tabular}{|c|}
$3^{\text {rd }}$ week \\
N (\%) \\
\end{tabular}}} & \multirow{2}{*}{\multicolumn{2}{|c|}{$\frac{6^{\text {th }} \text { weeks }}{\text { N }(\%)}$}} & \multirow{2}{*}{\multicolumn{2}{|c|}{\begin{tabular}{|c|} 
Pre-test \\
N $(\%)$
\end{tabular}}} & \multirow{2}{*}{\multicolumn{2}{|c|}{\begin{tabular}{|c|}
$3^{\text {rd }}$ week \\
N (\%) \\
\end{tabular}}} & \multicolumn{2}{|c|}{$6^{\text {th }}$ weeks } & & & \\
\hline & & & & & & & & & & & & & $(\%)$ & \multirow[b]{4}{*}{$.95 \mathrm{~ns}$} & & \\
\hline \multirow{3}{*}{$\begin{array}{c}\text { Pain or } \\
\text { discomfort } \\
\text { During } \\
\text { nocturnal bed } \\
\text { rest }\end{array}$} & & 3 & $\mathbf{1 0 . 0}$ & 15 & 50.0 & 28 & 93.3 & 3 & 34.4 & $\mathbf{0}$ & 0.0 & $\mathbf{0}$ & 0.0 & & \multirow{3}{*}{$\begin{array}{c}.001 \\
*\end{array}$} & \multirow{3}{*}{$\underset{* *}{0.001}$} \\
\hline & $\begin{array}{c}\text { Only on movement or in } \\
\text { certain positions }\end{array}$ & 19 & 63.3 & 13 & 43.3 & 2 & 6.7 & 20 & 66.7 & 19 & 63.3 & $\mathbf{1 0}$ & 33.3 & & & \\
\hline & With no movement & 8 & 26.7 & 2 & 6.7 & $\mathbf{0}$ & 0.0 & 7 & 33.3 & 11 & 36.7 & 20 & 66.7 & & & \\
\hline \multirow{3}{*}{$\begin{array}{c}\text { Morning stiffness } \\
\text { orregressive pain } \\
\text { after arising } \\
\end{array}$} & 1 minute or less & $\mathbf{1}$ & $\mathbf{3 . 3}$ & 13 & 43.3 & 28 & 93.3 & $\mathbf{1}$ & 3.3 & $\mathbf{0}$ & 0.0 & $\mathbf{2}$ & 6.7 & \multirow{3}{*}{$\begin{array}{c}.543 n \\
s\end{array}$} & \multirow{3}{*}{$\begin{array}{c}0.001 \\
* *\end{array}$} & \multirow{3}{*}{$\begin{array}{c}0.001 \\
* *\end{array}$} \\
\hline & $\begin{array}{c}\text { More than } 1 \text { but less } \\
\text { than } 15 \text { minutes }\end{array}$ & 17 & 56.7 & 17 & 56.7 & 2 & 6.7 & 20 & 70.0 & 15 & 50.0 & 7 & 23.3 & & & \\
\hline & 15 minutes or more & 12 & 04.4 & $\mathbf{0}$ & 0.0 & $\mathbf{0}$ & 0.0 & 9 & 26.7 & 15 & $\mathbf{5 0 . 0}$ & 21 & 70.0 & & & \\
\hline \multirow{4}{*}{ While ambulating } & \begin{tabular}{|c|} 
None \\
\end{tabular} & 2 & 6.7 & 16 & 53.3 & 28 & 93.3 & 3 & $\mathbf{1 0 . 0}$ & $\mathbf{0}$ & \begin{tabular}{|l|}
0.0 \\
\end{tabular} & $\mathbf{0}$ & 0.0 & \multirow{4}{*}{$\begin{array}{c}0.07 n \\
s\end{array}$} & \multirow{4}{*}{$\begin{array}{c}0.001 \\
* *\end{array}$} & \multirow{4}{*}{$\begin{array}{c}0.001 \\
* *\end{array}$} \\
\hline & \begin{tabular}{|c|}
$\begin{array}{c}\text { Only after ambulating } \\
\text { for } \\
\text { short distance }\end{array}$ \\
\end{tabular} & 12 & 40.0 & 13 & 43.3 & 2 & 6.7 & 5 & 16.7 & 4 & $\mathbf{1 3 . 3}$ & 5 & 16.7 & & & \\
\hline & $\begin{array}{c}\text { After initial ambulation } \\
\text { andincreasing with } \\
\text { continued ambulation }\end{array}$ & 14 & 46.7 & $\mathbf{1}$ & 3.3 & $\mathbf{0}$ & 0.0 & 22 & 73.3 & 24 & 80.0 & 21 & 70.0 & & & \\
\hline & $\begin{array}{c}\text { After initial ambulation, } \\
\text { not increasing }\end{array}$ & 2 & 6.7 & $\mathbf{0}$ & 0.0 & $\mathbf{0}$ & $\mathbf{0 . 0}$ & $\mathbf{0}$ & $\mathbf{0 . 0}$ & 2 & 6.7 & 4 & 13.3 & & & \\
\hline \multirow{2}{*}{$\begin{array}{l}\text { While getting up } \\
\text { from sitting } \\
\text { without the helps } \\
\text { of the arms } \\
\end{array}$} & \begin{tabular}{|l|} 
With difficulty \\
\end{tabular} & 7 & 23.3 & 11 & 36.7 & 25 & 83.3 & 4 & 13.3 & 2 & 6.7 & 2 & 6.7 & \multirow[b]{2}{*}{$\begin{array}{c}0.23 n \\
s\end{array}$} & \multirow[b]{2}{*}{$\begin{array}{c}.005 * \\
*\end{array}$} & \multirow[b]{2}{*}{$\underset{* *}{0.001}$} \\
\hline & Without difficulty & 23 & 76.7 & 19 & 63.3 & 5 & 16.7 & 26 & 86.7 & 28 & 93.3 & 28 & 93.3 & & & \\
\hline Total pain score & Mean \pm SD & \multicolumn{2}{|c|}{$4.83 \pm 1.599$} & \multicolumn{2}{|c|}{$2.26 \pm 1.52$} & \multicolumn{2}{|c|}{$.36 \pm .927$} & \multicolumn{2}{|c|}{$8.46 \pm 1.38$} & 5.73 & $=1.201$ & & $\pm \mathbf{1 . 1 4}$ & $\begin{array}{c}.931 n \\
\mathrm{~s}\end{array}$ & 0.001 & 0.001 \\
\hline
\end{tabular}

P1= pretest p.2= after 3 wks.p. $3=$ after 6 wks. significant difference $\mathrm{P}>0.05 \quad$ significant difference $\mathrm{P} \leq 0.05 \quad$ highly significant $\mathrm{p}<0.01$

Chi-Square Tests and one-way a nova testNs $=$ Non

Table 3: Reveals that highly significant difference between study and control group duringfollow in all items of pain and there was improvement in mean and SD for total means regarding pain. In the $3^{\text {rd }}$ week Mean \pm SD was $2.26 \pm 1.52$ in the study group and it was $5.73 \pm 1.201$ in the control group

Table4: Comparison between study and control groups regarding to the maximum distance walked after applying the quadriceps muscle strength exercise $\mathbf{n}=\mathbf{6 0}$

\begin{tabular}{|c|c|c|c|c|c|c|c|c|c|c|c|c|c|c|c|}
\hline \multirow{4}{*}{\begin{tabular}{|l|} 
Variable \\
Unlimited \\
\end{tabular}} & \multicolumn{6}{|c|}{ Study group } & \multicolumn{6}{|c|}{ Control group } & \multirow{3}{*}{ P1 } & \multirow{3}{*}{$\mathbf{P 2}$} & \multirow{3}{*}{ P3 } \\
\hline & \multirow{2}{*}{\multicolumn{2}{|c|}{\begin{tabular}{c|} 
Pre-test \\
N $(\%)$ \\
\end{tabular}}} & \multirow{2}{*}{\multicolumn{2}{|c|}{\begin{tabular}{|c|}
$3^{\text {rd }}$ week \\
$\mathrm{N}(\%)$ \\
\end{tabular}}} & \multirow{2}{*}{\multicolumn{2}{|c|}{$\begin{array}{c}6^{\text {th }} \text { weeks } \\
\text { N }(\%)\end{array}$}} & \multirow{2}{*}{\multicolumn{2}{|c|}{\begin{tabular}{|l} 
Pre-test \\
N $(\%)$
\end{tabular}}} & \multirow{2}{*}{\multicolumn{2}{|c|}{$\begin{array}{c}3^{\text {rd }} \text { week } \\
\text { N }(\%)\end{array}$}} & \multirow{2}{*}{\multicolumn{2}{|c|}{\begin{tabular}{|c|}
$6^{\text {th }}$ weeks \\
N $(\%)$
\end{tabular}}} & & & \\
\hline & & & & & & & & & & & & & & & \\
\hline & 0 & \begin{tabular}{|l|}
0.0 \\
\end{tabular} & 4 & 13.3 & 25 & 83.3 & $\mathbf{0}$ & 0.0 & $\mathbf{0}$ & \begin{tabular}{|l|}
0.0 \\
\end{tabular} & \begin{tabular}{|l}
0 \\
\end{tabular} & \begin{tabular}{|l|}
0.0 \\
\end{tabular} & \multirow{9}{*}{$\begin{array}{l}0.25 \\
3 \mathrm{~ns}\end{array}$} & \multirow{9}{*}{$\begin{array}{c}0.00 \\
1 * *\end{array}$} & \multirow{9}{*}{$\begin{array}{l}0.0 \\
1 *\end{array}$} \\
\hline $\begin{array}{l}\text { More than } 1 \mathrm{Km} \text {, but } \\
\text { Limited }\end{array}$ & 4 & 13.3 & 17 & 56.7 & 4 & 13.3 & 3 & 10.0 & $\mathbf{0}$ & 0.0 & $\mathbf{0}$ & 0.0 & & & \\
\hline $\begin{array}{l}\text { About a km (in about } \\
15 \mathrm{~min} \text { ) }\end{array}$ & 10 & 33.3 & 5 & 16.7 & 1 & 3.3 & 6 & 20.0 & $\mathbf{0}$ & 0.0 & 1 & 3.3 & & & \\
\hline \begin{tabular}{|l|} 
From 500-900m (in \\
about 8-15 min )
\end{tabular} & 4 & 13.3 & 2 & 6.7 & $\mathbf{0}$ & 0.0 & 10 & 33.3 & 2 & 6.7 & 1 & 3.3 & & & \\
\hline From 300-500m & 6 & 20.0 & 2 & 6.7 & $\mathbf{0}$ & 0.0 & 5 & 16.7 & 11 & 36.7 & 8 & 26.7 & & & \\
\hline From $100-300 \mathrm{~m}$ & 1 & 3.3 & $\mathbf{0}$ & 0 & $\mathbf{0}$ & 0.0 & 4 & 13.3 & 15 & 50.0 & 11 & 36.7 & & & \\
\hline Less than $100 \mathrm{~m}$ & 3 & 10.0 & $\mathbf{0}$ & $\mathbf{0}$ & $\mathbf{0}$ & 0.0 & 2 & 6.7 & 2 & 6.7 & 9 & 30.0 & & & \\
\hline $\begin{array}{l}\text { With one walking stick } \\
\text { or crutch }\end{array}$ & 2 & 6.7 & $\mathbf{0}$ & $\mathbf{0}$ & $\mathbf{0}$ & 0.0 & 0 & 0.0 & $\mathbf{0}$ & 0.0 & $\mathbf{0}$ & 0.0 & & & \\
\hline $\begin{array}{l}\text { With two walking } \\
\text { sticks or crutches }\end{array}$ & $\mathbf{0}$ & 0.0 & $\mathbf{0}$ & $\mathbf{0}$ & $\mathbf{0}$ & 0.0 & 0 & 0.0 & $\mathbf{0}$ & 0.0 & 0 & 0.0 & & & \\
\hline \begin{tabular}{|l} 
Total Maximum \\
distance walked (maywalk with \\
pain )
\end{tabular} & \multicolumn{2}{|c|}{$\begin{array}{c}3.23 \pm 1.79 \\
4\end{array}$} & \multicolumn{2}{|c|}{$1.36 \pm 1.03$} & \multicolumn{2}{|c|}{$.20 \pm .48$} & \multicolumn{2}{|c|}{$3.23 \pm 1.38$} & \multicolumn{2}{|c|}{$4.56 \pm .72$} & \multicolumn{2}{|c|}{$\begin{array}{c}4.86 \pm 1.00 \\
8\end{array}$} & $\begin{array}{c}1.00 \\
0 \\
\text { ns }\end{array}$ & $\begin{array}{c}0.00 \\
1 * *\end{array}$ & $\begin{array}{l}0.00 \\
1 \% *\end{array}$ \\
\hline
\end{tabular}

P1= pretest p.2= after 3 wks p.3= after 6 wks Chi-Square Tests and one way anova testNs $=$ Non significant difference $P>0.05 \quad$ significant difference $P \leq 0.05 \quad$ highly significant $\mathbf{p}<0.01$

Table 4: This table shows that no statistically significant difference between study and control group at the pre-test and statistically significance difference between study and control group regarding all items of Maximum distance walked during follow up after $6^{\text {th }}$ week and there were improvement in mean and SD for total means regarding Maximum distance walked $20 \pm .48, p \leq 0.05$. 


\section{daily activity}

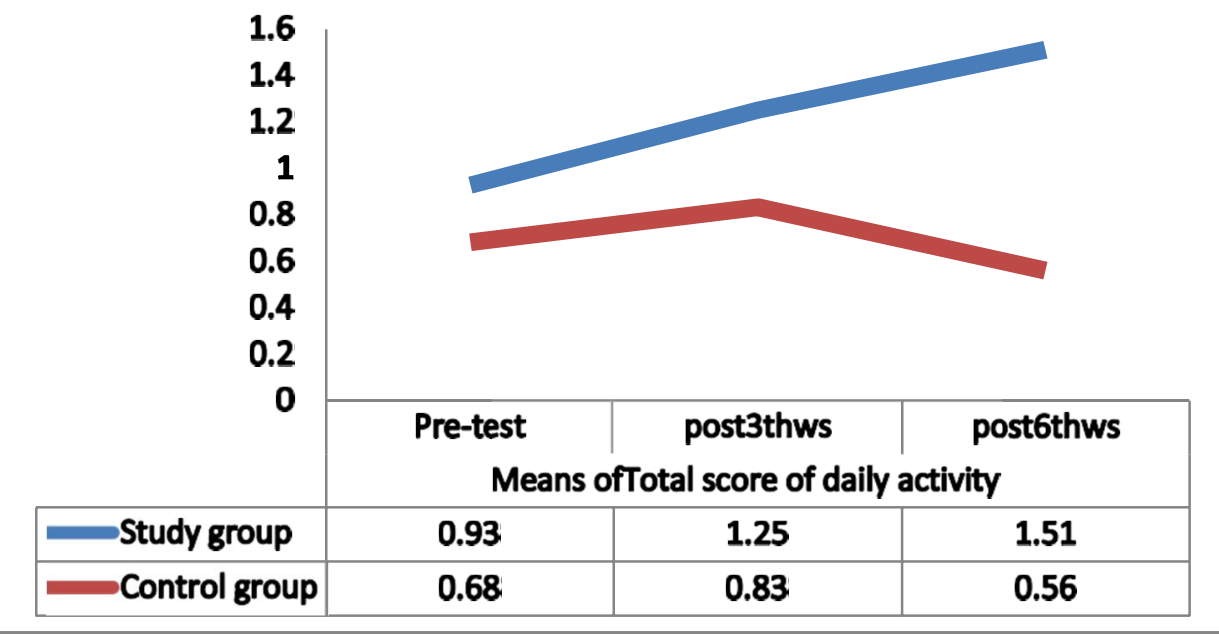

Fig.1: Means of daily activity between study and control group $n=60$

This Figure shows that no statically significant difference between study and control at pre-test and statically significance difference between study and control group regarding all items of Activities of daily living during follow up there were improvement in mean and SD for total means regarding Total score of daily activity. $\mathrm{p} \leq .05$

\section{Discussion:}

Osteoarthritis (OA) is the most common musculoskeletal condition (Rathwa et al., 2019). Arthritis in patients' knees affect gait because of three factors: pain, stiffness, and weakness. The same goes for arthritis in the feet. In addition, weakness in the muscles around the knee often occur in patients with arthritis, Fawole et al., (2020).

The aim of the present study was to determine effect of quadriceps muscle strength exercise on the patients with osteoarthritis at Aswan university hospital.

In my opinion quadriceps muscle strength exercise succeeded to improve joint mobility, muscle strength, distance walked level of activity and decrease joint stiffness and the level of pain. This reflects the desirable effect of exercise training on knee OA patients. It emphasized that exercise is important to enhance or maintains muscle strength, physical fitness, relieve the symptoms of knee OA and improve overall health.

Regarding the present and past history of the studied group: Unilateral Knee osteoarthritis (OA) affect left leg and Bilateral Knee Osteoarthritis affect both right and left in study and control group, Messier et al., (2016) found similar functional ability and perceived functional ability between unilateral and bilateral knee OA groups. In contrast, Aljehani et al., (2019) indicated that knee flexion during stance, and the external knee flexion moment were different between unilateral and bilateral knee OA. Unilateral OA patients presented with asymmetric gait whereas symmetry characterized bilateral OA gait

Studies providing insight into these questions have varied conclusions; one suggesting that unilateral and bilateral knee OA patients were functionally similar Messier et al., (2016) while another reporting that knee mechanics differs between the groups.

Regarding knee osteoarthritis assessment: The exciting study found that; there was statically significance difference between study and control group of Knee Osteoarthritis Assessment post application of quadriceps muscle strength exercises regarding reducing of pain, complete flexion and improving walking by maximum distance walked.

This agreed with Hauser \& Woldin, (2018) who documented that; the affected joint degenerates pain and restriction of movement often occur. Inflammation can also occur sometimes resulting in edema of the joint with OA. Treatment focuses on decreasing pain and improving movement.

Also, this result match with Nazari et al., (2019) who concluded that comparing to control had a statistically beneficial effect on range of motion, function and knee strength.

Our results matched with Nahas, (2019) who reported that; exercise, as part of a comprehensive arthritis treatment plan, can improve joint mobility, muscle strength, and overall physical conditioning, and help patient maintain a healthy weight. In the present study, the reduction in pain and disability in the experimental group may be attributed to increased quadriceps muscle strength and thereby improved stability, which leads to reduction of pain and disability Further, Aydoğdu et al., (2017) concluded in their study that exercise and physical agents can reduce pain and improve function and health status in patients with knee OA. Since pain and disability are interdependent, a reduction in one will cause a reduction in the other.

Regarding activities of daily living, this study showed that no statically significant difference between study and control pre-test and statically significance difference between study and control group regarding all items of Activities of daily living during follow up there were improvement in mean and SD for total means regarding Total score of daily activity.

This result in agreement with Walker et al., (2016) who demonstrated that isometric quadriceps exercises brought significant gains in strength of the quadriceps muscle in the experimental group after the 5 -week training program. In the between-group analysis, the improvement in strength in the experimental group was 33\% greater than that of the control group at the end of the training period. 
Our findings match with those obtained in previous studies that have demonstrated the benefits of isometric exercise in strength training by Kilinc et al., (2019) who compared the effect of isokinetic versus isometric exercise in patients with osteoarthritis of the knee. They reported that both groups showed significant improvement in muscle strength at the end of the trial. However, the isokinetic exercise group showed slightly greater improvement compared with the isometric exercise group. Similarly, Onigbinde et al., (2017) reported a significant increase in muscle strength after 8 weeks of isometric quadriceps exercise in patients with knee OA.

Regarding the correlation between pain, maximum distance and daily activity the present study illustrated that negative correlation between activity of daily living and maximum distance in study groups.

The results of the present study agreed with HerediaRizo et al., (2019) who showed that the 5week period of intervention brought about a significant reduction in knee pain and improvement in function in the experimental group at the 5 th week in addition to ,the significant reduction in pain and improvement in function in the experimental group may be attributed to improved quadriceps strength and therefore increase stability of the knee joint.

The findings are consistent with the findings of previous investigation of Çolak et al., (2017) who have reported that exercise can reduce pain and increase the functional abilities of OA patients. The Fitness Arthritis and Seniors Trial by Runhaar et al., (2019) reported a modest $8 \%$ to $10 \%$ improvement in pain and functioning scores as a result of 18 months of aerobic or resistance exercise among their sample of knee OA patients.

Further, Gilbert et al., (2018) found the same positive effects of exercise programs on pain and function. It is well documented in the literature that impaired quadriceps strength has been found to be the greatest single predictor of lower limb functional limitation. Further study done by Mazloum et al., (2018) concluded that quadriceps strengthening has beneficial effect on pain and function in patients with OA knee. The study done by Thakur et al., (2017) reported that subjects having stronger quadriceps strength had less knee pain and better physical function as compared with those with the least strength.

The researcher opinion that strong muscles stabilize the joints in a proper alignment, attenuate shocks that are transmitted to the joints and minimize the effect of impact by spreading the forces out over a greater area so it may be hypothesized that improvement in muscle strength is one of the main causes of reduced pain and disability.

In my opinion rehabilitating patients through exercise programs reduce the inflammatory process, decrease pain, prevent further joint damage, maintain and restore decreased muscle dysfunction.

It is recommended to see which one has more influence on the increase in muscle strength in a future study. Finally, the 6-week of isometric quadriceps exercise program for patients with knee OA showed beneficial effects on quadriceps muscle strength, pain, and improving functional ability.

Conclusion: Quadriceps muscle strength exercise effective in improvement condition of knee. reduce pain and improve movement.
Recommendations: Nurses should emphasis to provide a quadriceps muscle strength exercises on the patients with osteoarthritis

\section{References:}

(1) Ahamed D. (2014): Relationship between the radiological gradings and both functional performance and health related quality of life among women with knee osteoarthritis. Doctoral Thesis. Faculty of Nursing. Alexandria of University.

(2) Aljehani, M., Madara, K., Snyder-Mackler, L., Christiansen, C., \& Zeni Jr, J. A. (2019): The contralateral knee may not be a valid control for biomechanical outcomes after unilateral total knee arthroplasty. Gait \& posture, 70, 179-184

(3) Aydoğdu, O., Sari, Z., Yurdalan, S. U., \& Polat, M. G. (2017). Clinical outcomes of kinesio taping applied in patients with knee osteoarthritis: a randomized controlled trial. Journal of back and musculoskeletal rehabilitation, 30(5), 1045-1051

(4) Çolak, T. K., Kavlak, B., Aydoğdu, O., Şahin, E., Acar, G., Demirbüken, İ., .. \& \& Polat, M. G. (2017). The effects of therapeutic exercises on pain, muscle strength, functional capacity, balance and hemodynamic parameters in knee osteoarthritis patients: a randomized controlled study of supervised versus home exercises. Rheumatology international, 37(3), 399-407

(5) Fawole, H. O., Idowu, O. A., Abaraogu, U. O., Riskowski, J. L., Dell'Isola, A., Steultjens, M. P., \& Chastin, S. F. M. (2020). Factors associated with self-reported fatigue in hip and/or knee osteoarthritis: a systematic review. Rheumatology, 59(Supplement_2), keaa111-122

(6) Gilbert, A. L., Lee, J., Ehrlich-Jones, L., Semanik, P. A., Song, J., Pellegrini, C. A., ... \& Chang, R. W. (2018). A randomized trial of a motivational interviewing intervention to increase lifestyle physical activity and improve self-reported function in adults with arthritis. In Seminars in arthritis and rheumatism (Vol. 47, No. 5, pp. 732-740

(7) Hauser, R. A., \& Woldin, B. A. (2018). Joint Instability as the cause of chronic musculoskeletal pain and its successful treatment with prolotherapy. Anatomy, Posture, Prevalence, Pain, Treatment and Interventions of Musculoskeletal Disorders

(8) Heredia-Rizo, A. M., Petersen, K. K., Madeleine, P., \& ArendtNielsen, L. (2019). Clinical outcomes and central pain mechanisms are improved after upper trapezius eccentric training in female computer users with chronic neck/shoulder pain. The Clinical journal of pain, 35(1), 65-76

(9) Kilinc, S., Kabayel, D. D., \& Ozdemir, F. (2019). Comparison of the effectiveness of isokinetic exercise vs isometric exercise performed at different angles in patients with knee osteoarthritis. Isokinetics and Exercise Science, (Preprint), 1-12

(10) Lequesne, M. G. (1997). The algofunctional indices for hip and knee osteoarthritis. The Journal of Rheumatology, 24(4), 779-781.

(11) Litwic A, Edwards MH, Dennison EM, Cooper C . (2013): Epidemiology and burden of osteoarthritis. Br Med Bull.; 105: 185-199.

(12) Mazloum, V., Rabiei, P., Rahnama, N., \& Sabzehparvar, E. (2018). The comparison of the effectiveness of conventional therapeutic exercises and Pilates on pain and function in patients with knee osteoarthritis. Complementary therapies in clinical practice, 31, 343-348

(13) Messier, S. P., Beavers, D. P., Herman, C., Hunter, D. J., \& DeVita, P. (2016). Are unilateral and bilateral knee osteoarthritis patients unique subsets of knee osteoarthritis? A biomechanical perspective. Osteoarthritis and cartilage, 24(5), 807-813

(14) Nahas, R. M., Dos Santos, V. H., \& Alabarse, S. L. (2019). Physical Exercise Improves Quality of Life in Patients with Connective Tissue Disease. In Connective Tissue Disease-Current State of the Art. 376.

(15) Nazari, A., Moezy, A., Nejati, P., \& Mazaherinezhad, A. (2019). Efficacy of high-intensity laser therapy in comparison with conventional physiotherapy and exercise therapy on pain and function of patients with knee osteoarthritis: a randomized controlled trial with 12 -week follow up. Lasers in medical science, 34(3), 505-516

(16) Onigbinde, A. T., Ajiboye, R. A., Bada, A. I., \& Isaac, S. O. (2017). Inter-limb effects of isometric quadriceps strengthening on untrained contra-lateral homologous muscle of patients with knee osteoarthritis. Technology and Health Care, 25(1), 19-27 
(17) Rathwa, A., Prajapati, N., \& Deepak, A. N. (2019). To Compare the Effectiveness of Proprioceptive Circuit Exercises Versus Open Kinematics Chain Exercises on Pain and Improve Muscle Strength and Physical Function in Osteoarthritis Knee patients. Indian Journal of Physiotherapy \& Occupational Therapy, 13(1).

(18) Runhaar, J., Beavers, D. P., Miller, G. D., Nicklas, B. J., Loeser, R. F., Bierma-Zeinstra, S., \& Messier, S. P. (2019). Inflammatory cytokines mediate the effects of diet and exercise on pain and function in knee osteoarthritis independent of BMI. Osteoarthritis and cartilage, 27(8), 1118-1123

(19) Saad AY,El Shatby AM ,Sofar SM (2015) . Effect Of A Physical Training Program On The Functional Health Status Among Patients With Knee, Vol.4 ,Pp96 -108.

(20) . Schulze-Tanzil, G (2019) . Intraarticular Ligament Degeneration Is Interrelated with Cartilage and Bone Destruction in Osteoarthritis., 8, 990. [CrossRef].
(21) Sebbag E, Felten R, Sagez F (2019). The world wide burden of musculoskeletal diseases : asystematic analysis of the world Health organization Burden of diseases Database 78(6):844.8.

(22) Teixeira P. 2012: Self-Regulation, Motivation, and Psychosocial Factors In Weight Management.Journal Of Obesity; 2012(1):112.)

(23) Thakur, R., Mande, M., \& Mitra, M. (2017). Effectiveness of Quadriceps Strengthening Exercises in Reducing Extensor Lag, Pain and Improving Functional Activity in Patients of KneeOsteoarthritis. Website: www. ijpot. com, 11(2), 18

(24) Walker, S., Blazevich, A. J., Haff, G. G., Tufano, J. J., Newton, R. U., \& Häkkinen, K. (2016). Greater strength gains after training with accentuated eccentric than traditional isoinertial loads in already strength-trained men. Frontiers in physiology, 7, 149 .

(25) https://www.who.int/medicines/areas/priority_medicines/Ch6_12O steo.pdf 